\title{
An Empirical Study of Effective Capacity Throughputs in 802.11 Wireless Networks
}

\author{
Alan Davy*, Brian Meskill*, Jordi Domingo-Pascual ${ }^{\dagger}$ \\ *Waterford Institute of Technology, \\ Cork Road, Waterford, Ireland. \\ \{adavy, bmeskill\}@ @ssg.org \\ $\dagger$ Universitat Politècnica de Catalunya, \\ Barcelona, Spain. \\ jordi.domingo@ac.upc.edu
}

\begin{abstract}
Current literature defines the effective capacity of a wireless link as the maximum throughput that can be supported while meeting specific Quality of Service targets on packet delay. This metric can be harnessed for a wide variety of QoS control routines within wireless networks such as traffic optimisation and delay sensitive admission control and routing. However to date, no empirical evaluation of the effective capacity of 802.11 wireless links has been carried out. We present an empirical study of the effective capacity throughput of $\mathbf{8 0 2 . 1 1}$ wireless links under a number of network scenarios. We evaluate an analytical effective capacity model and compare the result with an empirical evaluation. We find that with an accurate measurement of the channel service delay, the effective capacity model can approximate the empirical measurement quite well. We also evaluate the relationship between the effective bandwidth of multimedia traffic and demonstrate that when the effective bandwidth exceeds the effective capacity threshold of a wireless link, the probability of QoS violations increases. We conclude that the effective capacity measurement is usable within an operational setting, and can lead to optimized utilization of bandwidth in a wide range of delay sensitive control operations.
\end{abstract}

\section{INTRODUCTION}

In recent years there has been a dramatic increase in the delivery of delay sensitive multimedia services over wireless networks. With the introduction of Quality of Service (QoS) provisioning support in standards such as 802.11n [1] and 3GPP QoS architecture for LTE [2], wireless networks now have the capability of offering QoS provisioning of delay sensitive services to end user devices. However there still remains the challenge of optimizing these QoS provisioning schemes to maximize utilization of resources while maintaining QoS targets. A major challenge in this area is the issue of accurately measuring the availability of resources over wireless links to meet the QoS requirements of delay sensitive services given the varying state of the wireless link.

The objective is to accurately estimate the maximum service rate that can be supported by a wireless link while meeting specific QoS bounds on packet delay. Current literature has termed this the effective capacity (EC) of a wireless link [3]. An accurate estimate of EC can ensure that QoS provisioning of multimedia traffic over a wireless link can be optimal with respect to the wireless link properties. As wireless channels are commonly shared mediums which are loss prone and fading, this can impact on the capacity of the wireless link over time. This may also be specific to the particular environmental context of operation (e.g. mobility, urban setting, neighbor nodes, cross traffic), and the coding and modulation techniques employed. When estimating the EC of a wireless link, all factors impacting capacity must be accounted for, which can be very challenging indeed.

Recent research [3] has proposed a method of modeling the EC of a wireless fading channel through analysis of the data link layer queue behavior. The authors have demonstrated that this model in theory can accurately provide statistical guarantees on QoS targets over wireless links. This model has spawned a wide range of theoretical research papers in the area of wireless resource management with a view to QoS provisioning of delay sensitive services. However to the best of our knowledge, no validation of this model has been carried out on IEEE 802.11 wireless links. We aim to evaluate this model for a range of 802.11 physical technologies and varying environmental conditions using the OPNET Modeler ${ }^{\mathrm{TM}}$ simulation software [4].

The objective of this paper is to first validate the theoretical effective capacity model for IEEE 802.11 wireless links, studying the impact of various assumptions within the EC model and then evaluate the EC capabilities and performance of the IEEE 802.11 wireless links under varying environmental contexts. We also evaluate the relationship between the bandwidth requirements of multimedia traffic and the EC of a wireless link. In this paper we carry out a simulation based analysis of effective capacity measurements over 802.11 wireless links, where we vary both the range of communication, the channel coding and modulation schemes, the number of nodes competing for the shared channel and also the level of cross traffic within the shared channel.

We compare the empirically collected packet delay distributions at the wireless data link layer to the predicted effective capacity model to evaluate the QoS provisioning capabilities of the wireless links. We find that under ideal conditions with accurate measurement of channel service delay, the effective capacity model proposed by [3] matches well to the empirical results. However as the level of channel interference increases 
the accuracy of the model can reduce to a margin of error of up to $9 \%$. In such a case a more in-depth empirical analysis of the data link layer queue is required to ensure an accurate measurement. We also find that when the EB of a multimedia traffic flow increases beyond the measured $\mathrm{EC}$ of a wireless link, the probability of QoS target violations increases.

The paper is organized as follows. Section II provides a discussion of related work in the area of EC estimation and usage and motivates the particular EC model under analysis. Section III provides an overview of the EC model. Section IV discusses the measurement methodology used to estimate the EC of a wireless link. Section V defines the experimental setup used for this evaluation. Section VI discusses the results attained and evaluates both the validity of the EC model, the impact various factors have on this measurement and an analysis of the relationship between EB of multimedia traffic and EC of a wireless link. Finally section VII will conclude the paper with a discussion and outline future work.

\section{RELATED WORK}

The meeting of statistical delay target in packet based communication is a fundamental requirement of delay sensitive services such as voice and video delivery. Within a wired network, there has been much research into understanding the resource requirements of various traffic types to ensure delay targets are met. The concept of effective bandwidth has been extensively studied in the past, for example in [5] and [6]. This concept captures the asymptotic stochastic behavior of queuing delay for a traffic source. Understanding the effective bandwidth requirements of traffic within a network can aid a wide range of management processes in the provisioning of QoS within a network, such as admission control [7] and network planning [8]. Active based measurement tools have also been proposed to measure the available bandwidth between two hosts within a wired network with the aim of improving end to end QoS provisioning [9].

More recent research has emerged that focuses on the QoS provisioning capabilities of a wireless link [3]. In contrast to the deterministic nature of capacity within a wired network, where components of delay across a link (e.g. transmission and propagation delay) are static, the capacity of a wireless link is considered non-deterministic. In this respect, deterministic QoS targets can not be guaranteed. Wu and Negi [3] proposed an EC model which determined the statistical probability of meeting QoS targets of packet delay across a wireless link. This model is based on the dual of the effective bandwidth model of a time-varying traffic source. The EC model captures the asymptotic stochastic behavior of queuing delay for a timevarying wireless link. By understanding these properties of the wireless link, one can appropriately manage traffic to ensure specific QoS targets on packet delay are met.

The effective capacity model proposed by $\mathrm{Wu}$ and Negi [3] has motivated a broad range of research papers investigating the provisioning of delay sensitive services over wireless links such as voice and video. The authors of [10],[11],[12] and [13] focus on using the EC model of a wireless link as the basis of a cross-layer approach to power and channel allocation management for delay sensitive services over wireless links.

The effective capacity model has also been used as a delay sensitive performance metric over different wireless MAC techniques. For example in [14],[15] and [16], the authors analyzed the EC of cognitive radio channels given varying primary and secondary user scenarios. The authors in [17] analyzed the EC capabilities of a time division downlink scheduling system. Delay sensitive routing within a wireless multi hop network was also studied in [18] where the QoS capabilities of the wireless links were modeled using the EC model. The diversity and utilization of the proposed EC model in delay sensitive wireless networking scenarios motivates the authors to choose this model as the bases of the evaluation of EC over 802.11 wireless links.

These papers are primarily theoretical in nature and model the wireless link using Rayleigh fading channel models [19]. However no papers have attempted to evaluate the model empirically over 802.11 wireless links. The objective of this paper is to focus on analyzing the EC of the IEEE 802.11 MAC layer for a variety of settings within a simulated environment, and to evaluate the effective capacity of the wireless links. We also evaluate the relationship between EB of multimedia traffic and the EC of wireless links, to demonstrate the impact on packet delay, motivating the appropriateness of such a metric for delay sensitive QoS control operations. In the following section, we present the necessary knowledge to understand the effective capacity theory and the proposed measurement methodology.

\section{EfFective CAPACITy Model}

The theory of EC was developed by Wu and Negi [3] to define a data link layer model of buffer overflow probability for wireless links. This theory is the dual of effective bandwidth, which has been studied for many years in the area of wired networks [5], [6]. As the dual of this theory, the EC defines the maximum arrival rate a wireless link can support while maintaining specific targets of packet delay.

It has been shown [3] that the EC of a queuing system with time varying service rate process $S(t)$ at time $t$, being supplied by a traffic source of constant rate $\mu$ is:

$$
E C(\theta)=-\lim _{t \rightarrow \infty} \frac{1}{t \theta} \log E\left[\gamma e^{-\theta S(t)}\right]
$$

Using the theory of large deviations, Wu and Negi demonstrates that the delay distribution of this service process satisfies:

$$
\operatorname{Pr}\left\{D(t) \geq D_{\max }\right\} \approx \gamma(\mu) e^{-\theta(\mu) D_{\max }}
$$

where both $\gamma(\mu)$ and $\theta(\mu)$ are functions of the constant source rate $\mu$. The justification for using a constant arrival rate in this model has been discussed in [20]. In summary, the authors demonstrate that if a constant arrival rate arrives at a queue system with a time varying service process, the effective capacity of the system can be measured through analysis of the system queue behavior. In equation (2), the parameters 
$\{\gamma(\mu), \theta(\mu)\}$ provide the statistical characterization of the queue behavior of a time varying service process being offered a constant arrival rate $\mu$.

Assuming that (2) holds at equality, the authors of [21] have shown that through estimating the expected queue size $E[Q(t)]$, expected utilization of the queue $E[S r(t)]$ and the average remaining service time of a packet $\tau$ of a given queue system being supplied with an arrival rate of $\mu$, the parameters $\gamma(\mu)$ and $\theta(\mu)$ can be measured as follows:

$$
\begin{aligned}
\frac{\gamma(\mu)}{\theta(\mu)} & =E[D(t)] \\
& =\tau E[\operatorname{Sr}(t)]+\frac{E[Q(t)]}{\mu}
\end{aligned}
$$

where $\operatorname{Sr}(t) \in\{0,1\}$ is the indicator as to whether a packet is in service or not at a given random time $t$ and $Q(t)$ is the size of the queue in bits at a random time $t . \tau$ in (4) is the average remaining service time for a packet in service as seen by an arriving packet (conditional on there being a packet in service). $D(t)$ is the total delay experienced by a packet combining queue delay with channel service delay at a random time $t$. We can estimate $\gamma(\mu)$, the channel utilization, by noting any instance the queue is empty:

$$
E[S r(t)]=\operatorname{Pr}(Q(t)>0)=\gamma(\mu)
$$

We can use equation (3) to obtain $\theta(\mu)$ as a function of delay and channel utilization through the following relationship.

$$
\theta(\mu)=\frac{\gamma(\mu)}{E[D(t)]}
$$

We can use equation (4) to obtain $\theta(\mu)$ as a function of the queue size, channel service delay and channel utilization, through the following relationship.

TABLE I

EFFECTIVE CAPACITy Model Notation

\begin{tabular}{c|l}
\hline Notation & Description \\
\hline \hline$\mu$ & Throughput rate of at traffic source. \\
$D_{\max }$ & Target upper bound on packet delay. \\
$S(t)$ & Amount of data serviced by the service process at time $[0, t)$. \\
$Q(t)$ & Amount of data in the queue waiting to be serviced at time \\
& {$[0, t)$.} \\
$D(t)$ & Delay experienced by a source packet at time $[0, t]$. \\
$S r(t)$ & An indicator at to whether a packet is in service or not at a \\
$\gamma$ & randomly chosen time $t$. \\
$\theta$ & Probability of a non-empty queue. \\
$E C(\theta)$ & QoS exponent. \\
$\tau$ & Effective capacity of a service process given $\theta$. \\
& The average remaining service time of a packet in service \\
$\hat{\gamma}$ & (assuming there is one). \\
$\hat{q}$ & A sample probability of a non-empty queue. \\
$\hat{\tau}$ & A sample measure of average queue length (in bits). \\
$\hat{\theta}$ & A sample measure of the average remaining service time of \\
$\varepsilon$ & An empet in service (assuming there is one). \\
Target QoS violation probability.
\end{tabular}

$$
\theta(\mu)=\frac{\gamma(\mu) \mu}{\mu \tau(\mu) \gamma(\mu)+E[Q(t)]}
$$

With a method of calculating both $\gamma(\mu)$ and $\theta(\mu)$ from equations (5) and (6) or (7) we can then substitute these values into the effective capacity model in equation (2) to estimate the probability of queue overflow given a particular packet delay target $D_{\max }$. The two options for calculating $\theta(\mu)$ depend on whether one has direct access to calculating the total delay experienced by a packet $D(t)$ or just access to the queue size $Q(t)$ at any given random time $t$. We now discuss practical methods of estimating the effective capacity model parameters.

\section{Estimation of EfFective CAPACity Model PARAMETERS}

The estimation of the EC parameters are based on observations at the data-link layer queue over a measurement interval $T$. The basic procedure is as follows, over interval $T$, a number of samples are taken, say $N$. At the $n^{t h}$ sampling interval, the following quantities are recorded: $S r_{n}$ the indicator of whether a packet is in service $\left\{S r_{n} \in\{0,1\}, Q_{n}\right.$ the number of bits in the queue, $D_{n}$ the total delay experienced by a packet including queue delay and contention delay, and $T_{n}$ the remaining service time of a packet in service, if one is in service. The following sample means are calculated:

$$
\begin{aligned}
\hat{\gamma} & =\frac{1}{N} \sum_{n=1}^{N} S r_{n} \\
\hat{q} & =\frac{1}{N} \sum_{n=1}^{N} Q_{n} \\
\hat{d} & =\frac{1}{N} \sum_{n=1}^{N} D_{n} \\
\hat{\tau} & =\frac{1}{\hat{\gamma} N} \sum_{n=1}^{N} T_{n} .
\end{aligned}
$$

Then $\hat{\theta}$ is calculated via 6 or 7 using:

$$
\begin{gathered}
\hat{\theta}=\frac{\hat{\gamma}}{\hat{d}} \\
\hat{\theta}=\frac{\hat{\gamma} \mu}{\mu \hat{\tau} \hat{\gamma}+\hat{q}} \approx \frac{\hat{\gamma} \mu}{\hat{\gamma} / 2+\hat{q}}
\end{gathered}
$$

As the remaining service time of a packet in service is quite difficult to measure in practice due to random contention delay, authors of [21] claim that $\hat{\tau}$ can be approximate with $1 / 2 \mu$. This implies that the remaining service time of a packet is a function of the arrival rate $\mu$. These equations make up the channel estimation algorithm used to estimate the EC model parameters $\{\gamma(\mu), \theta(\mu)\}$. They will be used to predict the Quality of Service provisioning capabilities of a time varying wireless channel by approximating (2) with:

$$
\operatorname{Pr}\left\{D(t) \geq D_{\max }\right\} \approx \hat{\gamma} e^{-\hat{\theta} D_{\max }} .
$$




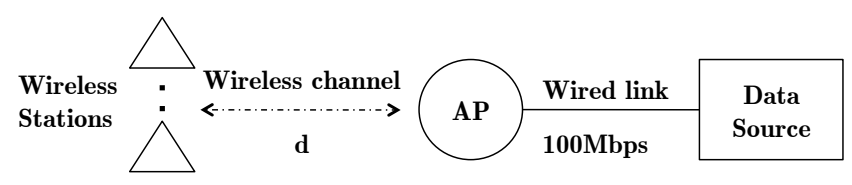

Fig. 1. Network Topology

We therefore wish to find an approximate arrival rate $\mu$ for a given $D_{\max }$ that will satisfy a given probability violation $\operatorname{target} \varepsilon$. The objective is to use the model to iteratively test different values of $\mu$ and to report when the probability of queue overflow is equal to a predefined probability violation target $\varepsilon$.

\section{Simulation SETUP}

We use the OPNET Modeler ${ }^{\mathrm{TM}}$ simulation environment [4] to both study the EC model and to evaluate the EC of 802.11 wireless links under varying network scenarios. We use a single hop topology as depicted in Fig. 1 for our network setup. The topology comprises of one data source node connected to a wireless access point (AP) using a wired link of throughout $100 \mathrm{Mbps}$. One or more wireless stations (WS) are connected to this AP using the specified wireless channel. The WSs are located equal distances from the AP requiring only one hop from the AP to receive data. All 802.11 MAC settings are configured to standard. The following 802.11 MACs are evaluated; Direct Sequence (802.11b), OFDM (802.11a).

Traffic is generated by the data source and is directed towards the WS through the AP to determine EC throughput. This traffic is UDP with a fixed payload of 1000 bytes. This is to ensure that each packet is small enough to avoid any fragmentation at the MAC layer. The throughout rate of the traffic is therefore controlled by the inter-departure time of each packet, which is uniformly distributed to ensure a constant bit rate throughout the simulation.

The AP model has been extended to collect the required samples to calculate the EC parameters as outline in section IV, namely the queue size $\left(Q_{n}\right)$ and service indicator $\left(S r_{n}\right)$. Samples are taken every 0.01 seconds for a duration of 200 seconds. To ensure the simulation has reached a steady state, we run each simulation for 400 seconds and use samples taken for the latter 200 seconds. This therefore means that a maximum of 20000 samples are used for the calculation of each parameter. As the EC model calls for the estimation of the remaining service time of a packet $\tau$, we do not collect this sample explicitly. We will however collect the total delay experienced by a packet at the wireless interface of the AP ,$\left(D_{n}\right)$. This is to evaluate the accuracy of the model with complete knowledge and to evaluate the assumption that the parameter $\tau$ can be estimated with the EC model.

For each experiment we use the samples collected to estimate the parameters $\hat{\gamma}$ and $\hat{\theta}$ using equations (8) and (12) or (13). Table. II depicts the set of parameters we will use within the experimental evaluation. Each experiment proceeds as follows: for a given set of parameters, an inter-departure time is chosen for the UPD constant bit rate traffic source. Traffic is then sent towards a single WS passing through the single AP. Samples are taken from the AP data link layer queue, and are processed offline. We present our proposed empirical measurement methodology within the following section.

TABLE II

EXPERIMENT PARAMETERS

\begin{tabular}{|r|l|}
\hline Distance (Meters) & 50,100 \\
Rates (Mbps) & 11,54 \\
Propagation Model & Free Space Path Loss \\
Transmitter Power (Watts) & 0.005 \\
Transmitter Antenna Gain (dBi) & 2 \\
Receiver Antenna Gain (dBi) & 2 \\
MAC Protocols & $802.11 \mathrm{~b}, 802.11 \mathrm{a}$ \\
Nodes & $1,3,5$ \\
Cross Traffic (bps) & $600 \mathrm{~K}, 1 \mathrm{M}, 3 \mathrm{M}, 5 \mathrm{M}$ \\
QoS delay targets & $D_{\max }=0.01 \mathrm{sec}, \varepsilon=0.0001$ \\
\hline
\end{tabular}

\section{A. Empirical Measurement Methodology}

To evaluate the accuracy of the EC model, we compare all estimates to a base set of empirical data. As the EC model aims to estimate the packet delay distribution through the use of the exponential function denoted in equation 2, we will directly collect the packet delay distribution $D_{n}$ from the AP within the simulation also at a sampling interval of 0.01 seconds. This is achieved by collecting the media access delay parameter of the wireless link within the OPNET modeler. OPNET provides this parameter directly and is defined as the total of queuing delay and contention delay of all packets transmitted by the MAC. From this sample set, we can create a cumulative distribution function (CDF) for the following $\operatorname{Pr}\left\{D_{n} \geq D\right\}$ where $D \in\left\{0 \ldots D_{\max }\right\}$. We can test our QoS target against both the CDF and the effective capacity model to determine the probability of packet delay being greater than the $D_{\max }$ target. At the end of each experiment, the collected data is analyzed to determine if the chosen traffic rate successfully meets the QoS target imposed. Fig. 2 depicts the search rule used to identify whether the EC has been found. Once found, the experiments are complete, however if the probability of violating the QoS target $D_{\max }$ is either higher or lower than the violation target $\varepsilon$ then the search will continue and a new simulation will be started with a new traffic inter-departure time. We employ a straight forward binary search algorithm to perform the search between the traffic rates of 0Mbps and the maximum theoretical rate of the physical technology within the experiment.

\section{RESUlts}

We began our evaluation of the EC model within an ideal scenario i.e. with only one WS connected to one AP and no competing cross traffic. This serves to determine the accuracy of the EC model in ideal conditions. The evaluation then progresses with the addition of various contending cross traffic scenarios at two different distances. Table. III depicts four 


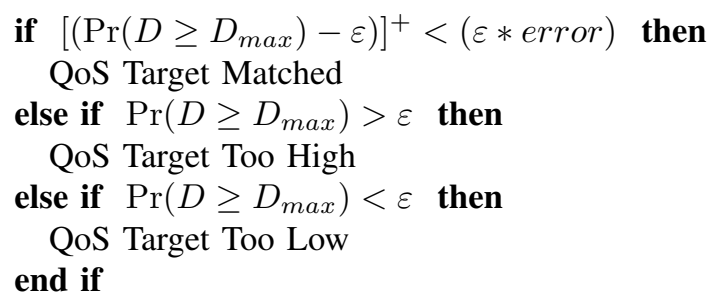

Fig. 2. Effective Capacity Search Rule

experimental results comparing the empirically collected data to the theoretical EC model with perfect estimation of channel service delay through the collection of parameter $D_{n}$.

TABLE III

EMPIRICAL EC VS EC MODEL

Experiment 1: 11Mbps, 802.11b links, distance of 50 Meters

\begin{tabular}{|l|c|c|c|}
\hline Nodes & Empirical EC (bps) & EC Model (bps) & Relative Error \\
\hline 0 & $4,867,181.301$ & $4,863,525.391$ & 0.075 \\
1 @ 3Mbps & $2,019,531.250$ & $2,094,726.563$ & 3.723 \\
3 @ 1Mbps & $1,933,593.750$ & $2,041,005.135$ & 5.555 \\
5 @ 1Mbps & $1,851,810.455$ & $2,006,927.013$ & 8.376 \\
\hline
\end{tabular}

Experiment 2: $11 \mathrm{Mbps}, 802.11 \mathrm{~b}$ links, distance of 100 Meters

\begin{tabular}{|l|c|c|c|}
\hline Nodes & Empirical EC (bps) & EC Model (bps) & Relative Error \\
\hline 0 & $4,864,868.164$ & $4,862,203.598$ & 0.055 \\
3 @ 1Mbps & $1,890,625.001$ & $2,062,500.000$ & 9.090 \\
5 @ 600Kbps & $3,137,390.137$ & $3,168,777.466$ & 1.000 \\
5 @ 1Mbps & $1,890,625.001$ & $2,065,185.547$ & 9.232 \\
\hline
\end{tabular}

Experiment 3: 54Mbps, 802.11a links, distance of 50 Meters

\begin{tabular}{|l|c|c|c|}
\hline Nodes & Empirical EC (bps) & EC Model (bps) & Relative Error \\
\hline 0 & $24,495,117.188$ & $24,476,907.670$ & 0.074 \\
3 @ 5Mbps & $9,755,859.375$ & $10,019,529.641$ & 1.267 \\
5 @ 3Mbps & $15,906,005.859$ & $15,905,778.140$ & 0.002 \\
5 @ 5Mbps & $9,755,859.375$ & $9,886,307.722$ & 1.337 \\
\hline
\end{tabular}

Experiment 4: 54Mbps, 802.11a links, distance of 100 Meters

\begin{tabular}{|l|c|c|c|}
\hline Nodes & Empirical EC (bps) & EC Model (bps) & Relative Error \\
\hline 0 & $24,528,076.172$ & $24,510,334.204$ & 0.072 \\
3 @ 5Mbps & $9,439,453.125$ & $9,559,277.985$ & 1.269 \\
5 @ 3Mbps & $15,609,375.000$ & $15,942,418.46$ & 2.133 \\
5 @ 5Mbps & $9,334,396.362$ & $9,729,655.449$ & 4.234 \\
\hline
\end{tabular}

The two physical technologies tested were $802.11 \mathrm{~b}$ and $802.11 \mathrm{a}$ with transmission rates of $11 \mathrm{Mbps}$ and $54 \mathrm{Mbps}$ respectively. We first evaluated the empirical data to the EC model with perfect channel service delay measurement at the lower rate of $11 \mathrm{Mbps}$. We found the model to be highly accurate in estimating the $\mathrm{EC}$ with a relative error of $0.075 \%$. EC was found to be about $4.86 \mathrm{Mbps}$ in both cases. In proportion to the maximum theoretical rate of $11 \mathrm{Mbps}$, the EC is a ratio of 0.442 approximately.

As cross traffic was introduced, there was a very slight increase in the relative error of approximately $1.2 \%$ between the empirical EC value and the EC model estimation. We also notice that as the cross traffic increases, the EC of the wireless link decreases in both the empirical data and the EC model, as can be seen in Table. III. This demonstrates that the EC model can accurately capture the impact cross traffic has on
TABLE IV

Effective Capacity Model Parameters Using $Q_{n}$ : Experiment 3

\begin{tabular}{|l|c|c|c|c|}
\hline Nodes & EC Model (bps) & $\hat{\theta}$ & $\hat{\gamma}$ & Relative Error \\
\hline 0 & $24,468,717.81$ & 697.704 & 0.9799 & 0.108 \\
3 @ 5Mbps & $9,927,246.094$ & 627.597 & 0.591 & 1.756 \\
5 @ 5Mbps & $9,876,983.643$ & 635.562 & 0.604 & 1.241 \\
\hline
\end{tabular}

the EC of a wireless link.

Fig. 3 depicts a semi-log scale graph of the empirical data of the packet delay distribution collected for Experiment 1 with 3 cross traffic nodes generating $1 \mathrm{Mbps}$ traffic each and sending this traffic towards the AP. The graph also depicts the distribution generated by the EC model at the same arrival rate. One can see that at this particular arrival rate, the empirical distribution shows that 0.001 of packets are delayed greater than 0.01 seconds. However the EC estimates that approximately 0.0004 of packets are delayed greater than 0.01 seconds. As can be seen in the results in Table. III, the EC model conservatively estimates the EC to be at 2,041,005.135 bps which is a relative error of $5.555 \%$.

At the higher transmission rate of 54Mbps, the EC model with perfect channel service delay measurements using the media access delay parameter $D_{n}$, achieved high accuracy in comparison to the empirical data with relative error as low as $0.072 \%$ with no interfering cross traffic. EC in both cases of 50 and 100 meters was found to be approximately $24.5 \mathrm{Mbps}$ which in comparison to the theoretical maximum is a ratio of 0.454. As cross traffic was introduced, the accuracy of the EC model estimation in comparison to the empirical data remained low with a relative error of up to $4.234 \%$.

At the higher transmission rate, however, solely using the queue size and the service indicator to calculate the EC model parameters, returned a high level of accuracy which is depicted in Table. IV for Experiment 3 parameters. We repeated the experiment for the distance of 100 meters also and found the results to be consistent. In addition to this, as cross traffic is introduced the accuracy of the EC estimates in comparison to the empirical data still remains low at about $1.7 \%$ relative error.

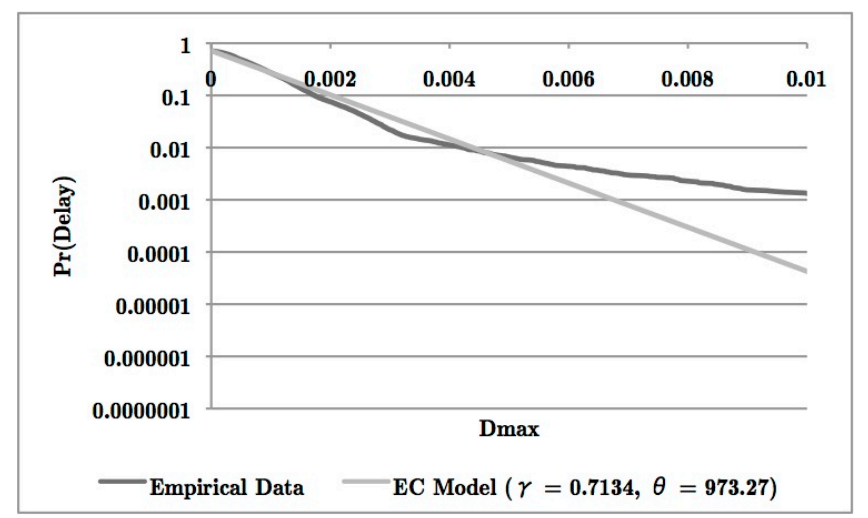

Fig. 3. Semi-Log Scale Empirical Data vs EC Model @ 1,933,593.75 bps for Experiment 1 


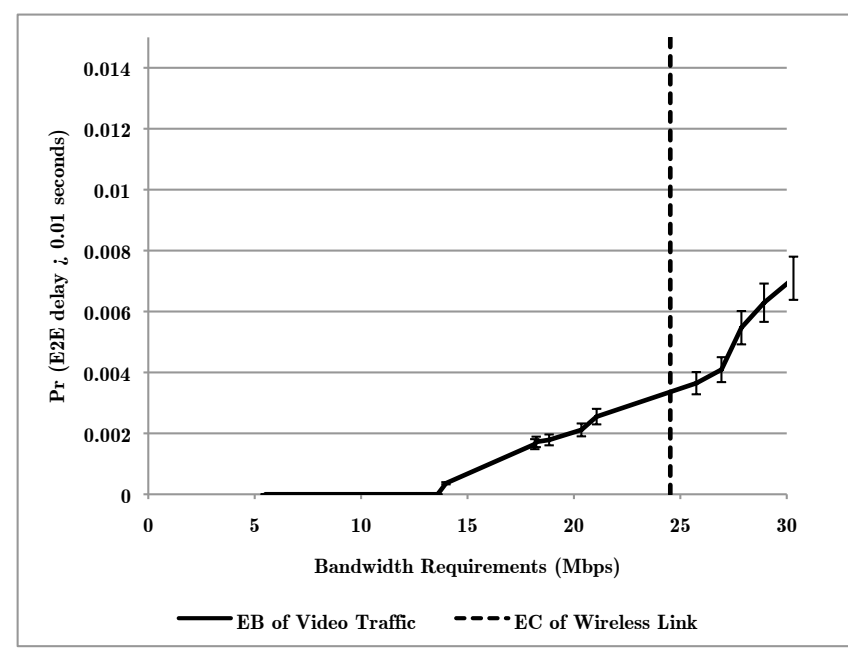

Fig. 4. Effective Bandwidth versus Probability of QoS Violation

\section{A. Comparison of Effective Bandwidth to Effective Capacity}

Given the measurement of EC has been recorded for each wireless scenario, we wish to evaluate the relationship between EC and the EB of a multimedia traffic flow across the AP towards the WS. The objective is to analyze the impact on end to end packet delay of multimedia traffic as the EB of the multimedia traffic increases above the EC threshold for the given wireless link scenario. The premise of the theory is that when the EB of the traffic increases beyond the EC threshold of the link, the likelihood of a violation in QoS targets on packet delay increases.

For the experiment, we use a video frame trace [?] which has been generated from an MPEG stream with a mean throughout of 770kbps. An increasing number of video flows were forwarded from the data source to the target WS. The delay between the data source and the access point was set to 0.0 seconds to ensure the end to end packet delay measurements collected within the OPNET Modeler ${ }^{\mathrm{TM}}$ only include the packet delay between the AP and the WS. The EB of the traffic flow is empirically estimated using the method outlined in ??. Packets traces are collected at the AP and processed through the EB algorithm for the specified QoS packet delay target of $10 \mathrm{~ms}$.

We focus on a single scenario within Experiment 3, where there is no competition between nodes. Fig. VI-A plots the EB of the traffic as the number of flows increase against the probability of violation the QoS packet delay target of $10 \mathrm{~ms}$. For each value on the plot, the experiment is executed 20 times with varying seed values. The plot shows that as the EB of the traffic increases beyond the EC threshold, the probability of violation increases. This demonstrates the accuracy of the EC measurement methodology and the impact on end to end packet delay.

\section{CONCLUSIONS}

We have presented an empirical analysis of the effective capacity throughput of 802.11 wireless links under varying network scenarios. We evaluated the proposed effective capacity model against the empirical data for a number of wireless network scenarios. With direct access to packet delay across a wireless link being available within the simulation environment, we determine that the effective capacity model delivers a high degree of accuracy ranging from $0.074 \%$ to $9.09 \%$ when compared to the empirical data. We also evaluated the relationship between the effective bandwidth of multimedia traffic and the effective capacity of a wireless link. We demonstrate that as the effective bandwidth requirements exceed the effective capacity threshold, the probability of QoS violations on packet delay increases. We therefore conclude that based on our analysis the effective capacity model is usable and the assumptions on estimation of channel service delay can yield a conservative estimates of effective capacity under varying network conditions. We plan to develop the effective capacity measurement methodology into a usable tool for wireless network delay sensitive control operations, such as admission control and routing.

\section{ACKNOWLEDGMENT}

This work has been funded by the Irish Research Council for Science, Engineering and Technology, co-funded by Marie Curie Actions under FP7 and the COST Action IC0703 Data Traffic Monitoring and Analysis (TMA) and supported by the OPNET Modeler software through the OPNET Technologies, Inc. University Program.

\section{REFERENCES}

[1] IEEE Computer Society, "Wireless lan media access control (mac) and plysical layer (phy) specifications," IEEE Standard for Information technology - Telecommuncations and information exchange between systems - Local and metrapolitan area networks - Specific requirements, no. 802.11n, October 2009.

[2] 3GPP, "Quality of service (qos) concept and architecture (release 10)," 3rd Generation Partnership Project: Technical Specification Group Services and System Aspects, vol. 10.0.0, no. TS 23.107, p. 41, 032011.

[3] D. Wu and R. Negi, "Effective capacity: a wireless link model for support of quality of service," Wireless Communications, IEEE Transactions on, vol. 2, no. 4, pp. $630-643$, july 2003.

[4] OPNET, "Discrete event smulation model library," OPNET Modeler ${ }^{T M}$, 2011. [Online]. Available: http://www.opnet.com/

[5] F. P. Kelly, S. Zachary, and I. Zeidins, Eds., Notes on effective bandwidth, vol. 4. Oxford University Press, 1996.

[6] G. Choudhury, D. Lucantoni, and W. Whitt, "Squeezing the most out of atm," Communications, IEEE Transactions on, vol. 44, no. 2, pp. 203 -217, feb 1996.

[7] A. Davy, D. Botvich, and B. Jennings, "Revenue Optimized IPTV Admission Control using Empirical Effective Bandwidth Estimation," IEEE Transactions on Broadcasting, vol. 54, issue 3 part 2, pp. 599 611, Sept 2008.

[8] A. Davy, B. Jennings, and D. Botvich, "QoSPlan: Process for QoSAware IP Network Planning Using Accounting Data and Effective Bandwidth Estimation," in Proc. of 2007 IEEE Global Telecommunications Conference (GLOBECOM), 2007, pp. 2690-2695.

[9] A. Cabellos-Aparicio, F. Garcia, and J. Domingo-Pascual, "A novel available bandwidth estimation and tracking algorithm," in Network Operations and Management Symposium Workshops, 2008. NOMS Workshops 2008. IEEE, April 2008, pp. 87 -94.

[10] X. Li and D. Wu, "Power control and channel allocation for real-time applications in cellular networks," WIRELESS COMMUNICATIONS \& MOBILE COMPUTING, vol. 8, no. 6, pp. 705-713, AUG 2008. 
[11] Y. Ma, H. Zhang, D. Yuan, and H.-H. Chen, "Adaptive power allocation with quality-of-service guarantee in cognitive radio networks," COMPUTER COMMUNICATIONS, vol. 32, no. 18, Sp. Iss. SI, pp. 19751982, DEC 152009.

[12] S. Vassilaras, "A cross-layer optimized adaptive modulation and coding scheme for transmission of streaming media over wireless links," WIRELESS NETWORKS, vol. 16, no. 4, pp. 903-914, MAY 2010.

[13] H. Zhang, Y. Ma, D. Yuan, and H.-H. Chen, "Quality-of-Service Driven Power and Sub-Carrier Allocation Policy for Vehicular Communication Networks," IEEE JOURNAL ON SELECTED AREAS IN COMMUNICATIONS, vol. 29, no. 1, pp. 197-206, JAN 2011.

[14] S. Akin and M. C. Gursoy, "Effective Capacity Analysis of Cognitive Radio Channels for Quality of Service Provisioning," IEEE TRANSACTIONS ON WIRELESS COMMUNICATIONS, vol. 9, no. 11, pp. 3354 3364, NOV 2010

[15] L. Musavian and S. Aissa, "Effective Capacity of Delay-Constrained Cognitive Radio in Nakagami Fading Channels," IEEE TRANSACTIONS ON WIRELESS COMMUNICATIONS, vol. 9, no. 3, pp. 1054-1062, MAR 2010

[16] L. Musavian, S. Aissa, and S. Lambotharan, "Effective Capacity for Interference and Delay Constrained Cognitive Radio Relay Channels," IEEE TRANSACTIONS ON WIRELESS COMMUNICATIONS, vol. 9, no. 5, pp. 1698-1707, MAY 2010.

[17] A. Balasubramanian and S. L. Miller, "The Effective Capacity of a Time Division Downlink Scheduling System," IEEE TRANSACTIONS ON COMMUNICATIONS, vol. 58, no. 1, pp. 73-78, JAN 2010.

[18] A. Abdrabou and W. Zhuang, "Statistical QoS Routing for IEEE 802.11 Multihop Ad Hoc Networks," IEEE TRANSACTIONS ON WIRELESS COMMUNICATIONS, vol. 8, no. 3, pp. 1542-1552, MAR 2009.

[19] B. Sklar, "Rayleigh fading channels in mobile digital communication systems .i. characterization," Communications Magazine, IEEE, vol. 35, no. 7 , pp. $90-100$, jul 1997.

[20] C.-S. Chang and J. Thomas, "Effective bandwidth in high-speed digital networks," Selected Areas in Communications, IEEE Journal on, vol. 13, no. 6, pp. $1091-1100$, aug 1995.

[21] B. L. Mark and G. Ramamurthy, "Real-time estimation and dynamic renegotiation of upc parameters for arbitrary traffic sources in atm networks," IEEE/ACM Trans. Netw., vol. 6, pp. 811-827, December 1998. [Online]. Available: http://dx.doi.org/10.1109/90.748091 